\title{
Treatment of Pulmonary Tumor Embolism from Choriocarcinoma: Extracorporeal Membrane Oxygenation as a Bridge through Chemotherapy
}

\author{
Jae Heun Chung, MD \\ Hye Ju Yeo, MD \\ Hyun Myung Cho, MD' \\ Jin Ook Jang, MD ${ }^{1}$ \\ Byung Min Ye, MD \\ Gun Yoon, MD2 \\ Dong Hoon Shin, $\mathrm{MD}^{3}$ \\ Dohyung Kim, $\mathrm{MD}^{4}$ \\ Woo Hyun Cho, MD 1
}

\begin{abstract}
A 22-year-old woman with a 1-month history of shortness of breath that was treated as a case of tuberculosis and pulmonary embolism was referred to the authors' hospital. Because of the hemodynamic instability in this patient, venoarterial extracorporeal membrane oxygenation (ECMO) was administered in the intensive care unit. She underwent a pulmonary embolectomy for the treatment of progressive circulatory collapse secondary to a pulmonary embolism. The histopathologic result was consistent with a metastatic choriocarcinoma. Despite the surgical management, persistent refractory cardiogenic shock occurred. Subsequently, the patient was treated with chemotherapy in the presence of ECMO and responded well to chemotherapy. She was discharged after 3 months. This case suggests that metastatic choriocarcinoma should be considered as a differential diagnosis in women of childbearing age presenting with a pulmonary embolism, and ECMO may be beneficial in patients with pulmonary embolism for bridging to surgical embolectomy and chemotherapy.
\end{abstract}

Departments of ${ }^{1}$ Internal Medicine, ${ }^{2}$ Obstetrics and Gynecology, ${ }^{3}$ Pathology, and ${ }^{4}$ Thoracic and Cardiovascular Surgery, Pusan National University Yangsan Hospital, Yangsan, Korea

\author{
Correspondence: Woo Hyun Cho, MD \\ Department of Internal Medicine, \\ Pusan National University Yangsan Hospital, \\ 20 Geumo-ro, Mulgeum-eup, \\ Yangsan 50612, Korea \\ Tel: 82-55-360-1414 \\ Fax: 82-55-360-1757 \\ E-mail: popeyes0212@hanmail.net \\ Received March 22, 2016 \\ Accepted June 6, 2016 \\ Published Online June 24, 2016
}

\section{Introduction}

Choriocarcinoma presenting as a pulmonary embolism is extremely rare. Because of the rarity of tumors of the pulmonary arteries, these tumors often are diagnosed inaccurately as venous thromboembolism or other more common disorders. This paper reports a case of a patient with a choriocarcinoma presenting as a massive pulmonary embolism $(\mathrm{PE})$, who required venoarterial (VA) extracorporeal mem- brane oxygenation (ECMO) support for bridging to a surgical embolectomy and chemotherapy.

\section{Case Report}

A 22-year-old woman (gravida 1, para 0) presented at the local hospital complaining of dyspnea. The patient described 

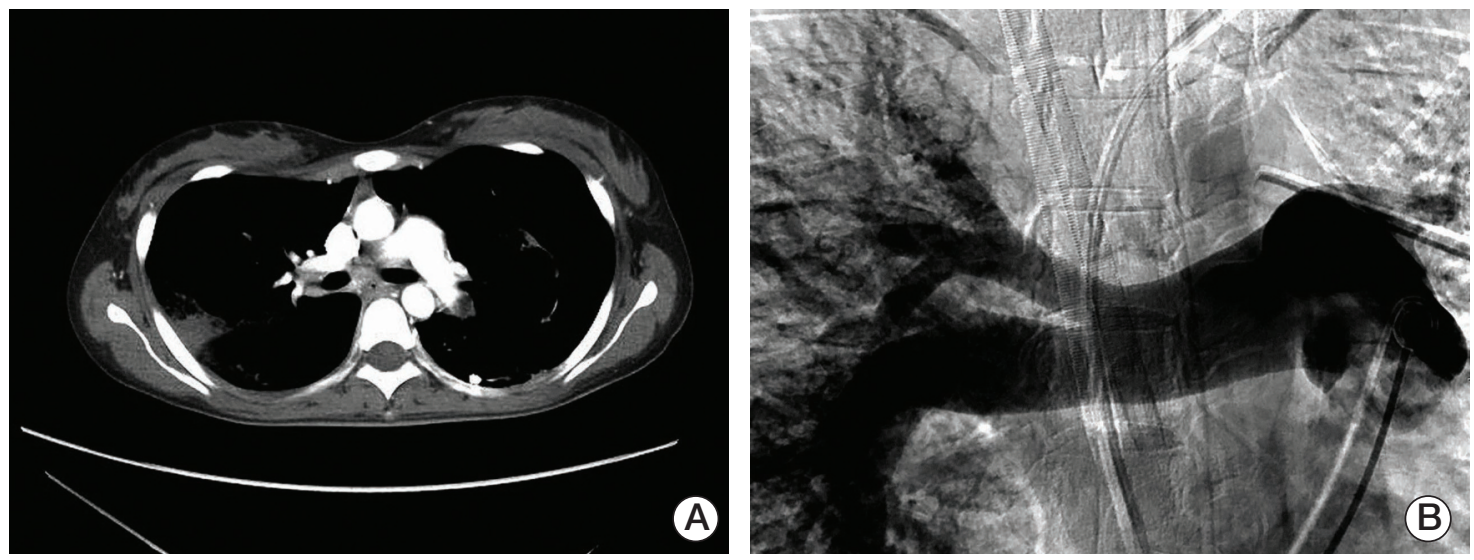

Fig. 1. (A) A chest enhanced computed tomography, demonstrating a filling defect in the left inferior pulmonary artery. (B) Pulmonary angiography, showing a large filling defect in the left pulmonary artery.
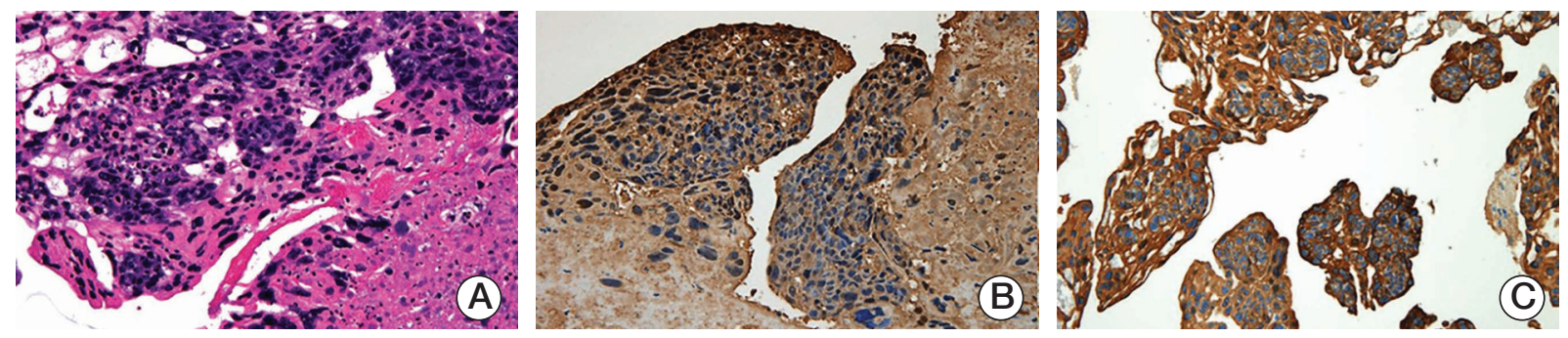

Fig. 2. (A) Diffuse sheets of cytotrophoblastic and syncytiotrophoblastic cells (H\&E staining, $\times 400$ ). (B) Tumor cells with diffuse positive cytoplasmic immunostaining for beta human chorionic gonadotropin $(\times 100)$. (C) Tumor cells with positivity for pan cytokeratin $(\times 40)$.

a cough, palpitation, and generalized myalgia. The patient had no prior medical history. She denied the use of tobacco products and said she consumed alcohol only occasionally. Her menstrual history revealed that she had experienced menarche at age 14, irregular cycles in the past, and occasional vaginal spotting in last two cycles. The chest radiographs revealed bilateral diffuse nodularity and a left pneumothorax. She was treated for pulmonary tuberculosis and pneumothorax with tuberculosis medications and the insertion of a chest tube; however, her symptoms worsened. A pulmonary thromboembolism was suspected, and chest enhanced computed tomography (CT) showed a filling defect in the left inferior pulmonary artery (Fig. 1A). Rivaroxaban was administered for the treatment of the pulmonary thromboembolism. Two weeks after starting treatment with rivaroxaban, the patient presented with aggravated dyspnea and massive hemoptysis and was urgently referred to the emergency department (ED).
Upon arrival at the ED, the patient was afebrile, with a heart rate of 138 beats per minute, blood pressure (BP) of $90 / 80 \mathrm{~mm} \mathrm{Hg}$, respiratory rate of 18 breaths per minute, and oxygen saturation of $100 \%$ in room air. She was alert and oriented. The chest examination for the breath sounds and auscultation revealed coarse crackles from the right lung and no sounds from the left.

The arterial blood gas levels under oxygen mask inhalation (5 L/min) were as follows: $\mathrm{pH}, 7.51 ; \mathrm{PaO}_{2}, 81 \mathrm{~mm} \mathrm{Hg}$; and $\mathrm{PaCO}_{2}, 25 \mathrm{~mm} \mathrm{Hg}$. Her hemoglobin level was $13.1 \mathrm{~g} / \mathrm{dL}$, white blood cell count was $7,520 / \mathrm{mL}$, and platelet count was $273,000 / \mathrm{mL}$. The D-dimer was $2.72 \mu \mathrm{g} / \mathrm{mL}$, and the troponin level was elevated slightly, at $0.01 \mathrm{ng} / \mathrm{mL}$. Her chest radiograph revealed a normal cardiac field, diffuse nodularity in both lung fields, and a pneumothorax with chest tubing in the left lung zone. While repositioning the chest tube into the pneumothorax, she became mentally confused, and the laboratory results showed a partial pressure of oxygen $\left(\mathrm{pO}_{2}\right)$ of 
$38 \mathrm{~mm} \mathrm{Hg}$. She was intubated, started on mechanical ventilation, and admitted to the intensive care unit for further evaluation.

On the second day after admission, her mean BP was decreased gradually. The patient remained hypotensive despite the intravenous fluid boluses that escalated the inotropic infusion with the maximum dose. VA ECMO was initiated to restore hemodynamic stability.

An echocardiogram revealed a severe left ventricular systolic dysfunction with an ejection fraction of $29 \%$ and a D-shaped left ventricle. Bronchoscopy revealed diffuse alveolar hemorrhage but no evidence of an endobronchial lesion. Therefore, the administration of rivaroxaban therapy was stopped. Pulmonary angiography was performed and showed a large filling defect in the left pulmonary artery (Fig. 1B). The patient underwent a pulmonary embolectomy in the presence of ECMO. At the site of the main pulmonary artery near its opening to the left pulmonary artery, a whitish tumor embolus was found in the left pulmonary artery. The tumor embolus was removed to the extent possible. Histologically, the pulmonary tumor embolism specimen revealed a malignancy consistent with choriocarcinoma (Fig. 2).

After a diagnosis of choriocarcinoma was made, the serum beta human chorionic gonadotropin (hCG) level was measured, and a gynecologic examination was performed. The serum beta hCG values were $260,000 \mathrm{mIU} / \mathrm{mL}$. A metastatic workup revealed no lesions in the liver, spleen, kidney, pelvic cavity, and brain (International Federation of Gynecology and Obstetrics [FIGO] stage III, FIGO prognostic score 6). Although she was under an ECMO insertion state and her platelet level was below $75,000 / \mu \mathrm{L}$, chemotherapy was started with the following conventional regimen after receiving fully informed consent: etoposide, $100 \mathrm{mg} / \mathrm{m}^{2}$ on days 1 and 2 ; methotrexate, $100 \mathrm{mg} / \mathrm{m}^{2}$ via intravenous bolus on day 1 and $200 \mathrm{mg} / \mathrm{m}^{2}$ via intravenous infusion over 12 hours on day 1 ; actinomycin D, $0.5 \mathrm{mg}$ via intravenous bolus on day 1 and 2; cyclophosphamide, $600 \mathrm{mg}$ via intravenous infusion on day 8 ; and vincristine, $1.0 \mathrm{mg} / \mathrm{m}^{2}$ on day 8 (EMA-CO). After the initial chemotherapy, her beta hCG level decreased to $2,296 \mathrm{mIU} / \mathrm{mL}$, and the ECMO was removed as her heart function was restored. The second cycle of chemotherapy was postponed because of a poor general condition and grade 4 neutropenia. After a 2-month period of chemotherapy, the follow-up CT showed dissolved emboli in the pulmonary artery. The patient was discharged from hospital with a normal beta hCG level.

\section{Discussion}

Choriocarcinoma is a malignant, gestational trophoblastic neoplasm that can occur after a molar hydatidiform mole, ectopic pregnancy, abortion, and even normal pregnancy. Choriocarcinomas spread hematogenously to the lungs, resulting in PE, pulmonary hypertension, or acute respiratory distress syndrome. Although the prognosis for metastatic gestational choriocarcinoma to the lungs (stage III) is good, the rarity of a PE can result in a misdiagnosis, and the patient might be treated for more common diseases, such as pneumonia and tuberculosis $[1,2]$. In the present case, the patient also was diagnosed incorrectly with tuberculosis and a venous pulmonary thrombosis. In the case of fertile women presenting with dyspnea, cough, and hemoptysis, it is essential to have a suspicion of a tumor embolism. Watanabe et al. [3] emphasized the importance of considering choriocarcinoma and measuring the serum beta hCG levels when evaluating fertile women presenting with pulmonary emboli or pulmonary artery hypertension. Therefore, it is recommended that the serum beta hCG level be performed as a diagnostic test in women of reproductive age to differentiate the causes of a PE.

Patients presenting with massive PE complicated by right ventricular failure and cardiogenic shock have high shortterm mortality (>25\%) [4]. The hemodynamic instability hinders a clinical evaluation including chest CT scan and pulmonary angiography. With regard to treatment, urgent thrombolysis or surgical approach is mandatory; however, these treatments might be limited because of refractory hypoxia and shock. VA ECMO could be an optional strategy in a patient with a massive $\mathrm{PE}$ as a bridge to further diagnostic work-up and therapeutic interventions. Chowdhury et al. [5] reported the use of VA ECMO as an adjunct to thrombolytic therapy for progressive circulatory collapse secondary to a massive acute PE.

As in other cases, the patient remained hypotensive despite intravenous fluid boluses, in which inotropic infusion was escalated escalating to the maximum dose. To perform diagnostic workup and therapeutic interventions, such as embolectomy and pulmonary angiogram, ECMO is essential to maintain hemodynamic stability.

To the best of the authors' knowledge, only a few cases of a pulmonary embolectomy to remove tumor emboli from choriocarcinoma have been reported. On the other hand, this is a rare case of a patient with a metastatic gestational choriocarcinoma who presented with a pulmonary artery tumor embolism by employing ECMO and pulmonary embolectomy followed by chemotherapy.

According to Worku et al. [6], a patient with lymphoma developed cardiogenic shock at the time of chemotherapy 
initiation. ECMO was instituted to restore the hemodynamic stability, and to bridge the patient to chemotherapy until the tumor burden could be reduced effectively. In this study, the patient experienced persistent cardiogenic shock despite the surgical embolectomy. Generally, chemotherapy is not recommended routinely in a patient under ECMO because of its adverse effects, such as neutropenia or thrombocytopenia. Despite this, the choriocarcinoma responded very well to the chemotherapeutic agent initiated, and the patient under VA ECMO responded positively, with serum beta hCG falling to within the normal levels. Therefore, although the prolonged use of ECMO would increase the risk of morbidity, including thrombotic and hemorrhagic complications, its adequate application allows the patient to achieve benefits as an effective bridging therapy, while minimizing complications.
These results suggest that although metastatic gestational choriocarcinoma is rare, it should be considered in a differential diagnosis of fertile women presenting with a massive PE. In addition, ECMO may be used as an adjunct to bridge to embolectomy and chemotherapy as soon as possible in a patient with hemodynamic instability, because of the potential for a good response of the choriocarcinoma to the chemotherapy.

\section{Conflicts of Interest}

Conflict of interest relevant to this article was not reported.

\section{References}

1. Khakural P, Shrestha KR, Sapkota R, Shrestha UK. An unusual case of pulmonary embolism. J Surg Case Rep. 2015;2015: rjv006.

2. Yang L, Xu P, Xi M, Wang H. FIGO stage IV gestational choriocarcinoma misdiagnosed as pulmonary tuberculosis: a case report. Oncol Lett. 2015;10:1924-6.

3. Watanabe S, Shimokawa S, Sakasegawa K, Masuda H, Sakata R, Higashi M. Choriocarcinoma in the pulmonary artery treated with emergency pulmonary embolectomy. Chest. 2002;121:654-6.

4. Darze ES, Latado AL, Guimaraes AG, Guedes RA, Santos AB, de Moura SS, et al. Acute pulmonary embolism is an independent predictor of adverse events in severe decompensated heart failure patients. Chest. 2007;131:1838-43.

5. Chowdhury MA, Moza A, Siddiqui NS, Bonnell M, Cooper CJ. Emergent echocardiography and extracorporeal membrane oxygenation: Lifesaving in massive pulmonary embolism. Heart Lung. 2015;44:344-6.

6. Worku B, DeBois W, Sobol I, Gulkarov I, Horn EM, Salemi A. Extracorporeal membrane oxygenation as a bridge through chemotherapy in B-cell lymphoma. J Extra Corpor Technol. 2015;47:52-4. 